\title{
Characterizing the 18 April 2002 storm-time sawtooth events using ground magnetic data
}

\author{
C. Robert Clauer, ${ }^{1}$ Xia Cai, ${ }^{1}$ Daniel Welling, ${ }^{1}$ Anna DeJong, ${ }^{1}$ and Michael G. Henderson ${ }^{2}$ \\ Received 22 February 2005; revised 20 September 2005; accepted 11 January 2006; published 22 April 2006.
}

[1] Beginning during the last half of the main phase and extending for over 12 hours, the 18 April 2002 magnetic storm is dominated by sawtooth oscillations. We examine the ground magnetic signatures of these events at both high latitudes and low latitudes. The first immediate observation is that the magnetic signatures from one sawtooth to another can show considerable variation. Thus the current systems associated with these events show considerable variation in location, pattern, and strength from one oscillation to the next. The characteristics of the magnetic disturbance for each sawtooth has little to distinguish it from the typical substorm expansion magnetic disturbance field except that some of the sawteeth disturbance profiles are broader in local time than observed during typical substorms. The injection region determined from the energetic particle flux measurements at geostationary orbit is generally broad, extending across the entire tail and past the terminators into the dayside. The injection region is usually wider than the magnetic disturbance profile at the peak of the dipolarization. There appears to be no relationship between the magnetic disturbance profile and the location and strength of the injection boundary. Finally, sawtooth events appear to be driven by a steady solar wind driver and to develop during a period of already enhanced ring current.

Citation: Clauer, C. R., X. Cai, D. Welling, A. DeJong, and M. G. Henderson (2006), Characterizing the 18 April 2002 storm-time sawtooth events using ground magnetic data, J. Geophys. Res., 111, A04S90, doi:10.1029/2005JA011099.

\section{Introduction}

[2] It has recently been recognized that strong magnetospheric driving can lead to a phenomena called global sawtooth oscillations. J. R. Borovsky et al. (The solar-wind driving of global sawtooth oscillations and periodic substorms: What determines the periodicity, submitted to Annales Geophysicae, 2006, hereinafter referred to as Borovsky et al., submitted manuscript, 2006) describe sawtooth events as global, quasi-periodic, large-amplitude oscillations in the flux of energetic particles measured at geostationary orbit. They derive their name because of their shape in the energetic particle flux data consisting of a series of slow flux decreases followed by rapid increases thus resembling the teeth of a saw blade. The sawtooth shape is particularly well defined in the high-energy proton channels [Henderson, 2004]. The period of the oscillation is between 2 and 4 hours and the rapid flux enhancement portion of the oscillation lasts between 5 and $15 \mathrm{~min}$.

[3] The sawtooth-like particle flux variations are indicative of changes in the morphology of the magnetic field in the inner magnetosphere undergoing strong stretching and then rapid dipolarization. While this is typical behavior on the nightside for substorm disturbances, the unique aspect of sawtooth events is that this behavior is observed across

\footnotetext{
${ }^{1}$ University of Michigan, Ann Arbor, Michigan, USA.

${ }^{2}$ Los Alamos National Laboratory, Los Alamos, New Mexico, USA.
}

Copyright 2006 by the American Geophysical Union. 0148-0227/06/2005JA011099 the entire nightside and occasionally past the dawn or dusk terminators into the dayside. Sawtooth oscillations are large events that may represent a global mode of energy dissipation. Sawtooth events have similarities and differences with periodic substorms and a debate has emerged as to whether global sawtooth oscillations are simply an extreme form of substorm or whether they merit a category to themselves [Henderson, 2004; Huang et al., 2003; Borovsky et al., submitted manuscript, 2006]. The phenomena typically described as periodic substorms, for example in the work of Borovsky et al. [1993], tend to occur during high-speed streams when the interplanetary magnetic field is fluctuating northward and southward for short intervals during long periods and the magnetic field distortions are thought to be smaller and localize in the nightside (though this has not been quantitatively demonstrated as yet).

[4] Sawtooth events occur during storms when the ring current is enhanced, and they appear to be driven by steady moderate to strong southward IMF conditions (particularly during magnetic cloud interactions with the magnetosphere). Often the plasma sheet appears to be unusually close to the Earth at the time of sawtooth events, suggesting that the plasma convection in the inner magnetosphere is unusually strong. Sawtooth oscillations occur during some, but not all, geomagnetic storms and it still remains to determine the storm-time conditions required to produce these phenomena. Since sawtooth oscillations are generally observed during rather steady solar wind driving, their relationship to steady magnetospheric convection (SMC) intervals is a question to consider. A popular paradigm is 
Table 1. Magnetic Observatories

\begin{tabular}{|c|c|c|c|c|c|c|}
\hline Station Name & $\begin{array}{l}\text { Station } \\
\text { Code }\end{array}$ & $\begin{array}{c}\text { Geographic } \\
\text { Latitude }\end{array}$ & $\begin{array}{c}\text { Geographic E } \\
\text { Longitude }\end{array}$ & $\begin{array}{c}\text { CGM } \\
\text { Latitude }\end{array}$ & $\begin{array}{c}\text { CGM } \\
\text { Longitude }\end{array}$ & UT MLMidnight \\
\hline Trelew & TRW & -43.3 & 294.7 & -29.59 & 4.96 & 4.14 \\
\hline San Juan & SJG & 18.4 & 293.9 & 28.36 & 10.45 & 4.21 \\
\hline Kourou & $\mathrm{KOU}$ & 5.1 & 307.4 & 8.87 & 23.48 & 3.19 \\
\hline Guimar & GUI & 28.3 & 343.6 & 14.11 & 60.68 & 0.48 \\
\hline Ebro & EBR & 40.8 & 0.5 & 33.96 & 76.19 & 23.23 \\
\hline Hermanus & HER & -34.4 & 19.2 & -42.34 & 82.57 & 23.79 \\
\hline L'Aquila & AQU & 42.4 & 13.3 & 36.24 & 87.35 & 22.39 \\
\hline Hartebeestohoek & HBK & -25.9 & 27.7 & -36.07 & 95.04 & 23.22 \\
\hline Lourenco Marques & LMM & -25.9 & 32.6 & -35.96 & 99.95 & 22.93 \\
\hline Istanbul Kandilly & ISK & 41.1 & 29.1 & 35.53 & 101.59 & 21.43 \\
\hline Qsaybeh & QSB & 35.6 & 33.9 & 29.54 & 105.74 & 21.18 \\
\hline Antananarivo & TAN & -18.9 & 47.5 & -28.83 & 116.66 & 21.95 \\
\hline Alibag & $\mathrm{ABG}$ & 18.6 & 72.9 & 11.86 & 145.27 & 18.83 \\
\hline Lanzhou & $\mathrm{LZH}$ & 36.1 & 103.8 & 30.45 & 176.03 & 17.14 \\
\hline Learmonth & LRM & -22.2 & 114.1 & -33.28 & 185.54 & 16.73 \\
\hline Beijing Ming Tombs & BMT & 40.3 & 116.2 & 34.59 & 188.76 & 16.45 \\
\hline Kanoya & KNY & 31.4 & 130.9 & 24.66 & 202.83 & 15.59 \\
\hline Kakioka & KAK & 36.2 & 140.2 & 29.25 & 211.8 & 15.06 \\
\hline Chichijima & CBI & 27.1 & 142.3 & 19.84 & 213.43 & 14.91 \\
\hline Memambetsu & MMB & 43.9 & 144.2 & 37.08 & 215.49 & 14.88 \\
\hline Apia & API & -13.8 & 188.2 & -15.56 & 262.46 & 10.39 \\
\hline Honolulu & $\mathrm{HON}$ & 21.3 & 202. & 21.4 & 269.83 & 11.13 \\
\hline Papeete & PPT & -17.5 & 210.4 & -16.6 & 285.17 & 8.88 \\
\hline Fresno & FRN & 37.1 & 240.3 & 43. & 303.55 & 8.47 \\
\hline Tucson & TUC & 32.3 & 249.2 & 39.89 & 314.41 & 7.75 \\
\hline Del Rio & DLR & 29.5 & 259.1 & 38.88 & 326.27 & 6.96 \\
\hline Bay St. Louis & BSL & 30.4 & 270.6 & 41.41 & 340.7 & 6.05 \\
\hline
\end{tabular}

that substorms result because of imbalance between dayside and nightside reconnection. Enhanced dayside reconnection transports flux to the tail (loading), the plasma sheet thins, and an instability initiates a near-Earth reconnection region that converts the stored magnetic energy to particle kinetic energy (unloading). If, however, the enhanced dayside reconnection rate is steady for a long period of time, the nightside reconnection rate can adjust to match the dayside rate and enhanced steady magnetospheric convection results. The existence of sawtooth oscillations during steady driving suggests that some driving conditions result in a sort of driven oscillation rather than a steady convection. Thus the relationship between the driven sawtooth periodic behavior, substorms, and the periodic substorms that develop during high-speed streams where frequent short southward IMF intervals cause an accumulation of flux in the tail that eventually unloads, requires a detailed examination.

[5] In this paper, we examine the magnetic disturbance field measured on the ground to determine the characteristics of the sawtooth disturbance. While satellites provide a good in situ description of the disturbance, they are severely limited by the localization of the observations. Arrays of ground instruments provide a synergy by enabling a global description of the disturbance field and we shall use both high-latitude and low-latitude ground magnetic data to quantify the storm-time disturbance field during the sawtooth oscillations observed during the 18 April 2002 magnetic storm. Kitamura et al. [2005] also examine ground magnetic data during these same sawtooth events.

[6] To better capture both the temporal and spatial development of the ring current during storms, we have extended the analysis procedure developed by Clauer and McPherron [1974a, 1980] to the investigation of several large geomagnetic storms and have applied the analysis to the 17-24 April 2002 storm period. The analysis is based upon the use of data from a worldwide longitudinal chain of midlatitude magnetic observatories.

[7] Midlatitude magnetic data have a long history of use in the study of storms and substorms. For example, Kamide and Fukushima [1971] used the $\mathrm{H}$ variation at midlatitudes to calculate the parameters of simple line current models of the symmetric and partial ring current. Clauer and McPherron [1980] examined midlatitude measurements to investigate the association of partial ring current development with isolated substorms, finding that the partial ring current may begin to develop prior to the onset of the substorm expansion, but a southward turning of the interplanetary magnetic field consistently precedes the onset of partial ring current development. Clauer et al. [2003], utilizing midlatitude magnetic measurements to study the relationship between substorms and storms, show that the development of the storm-time ring current is not the result of a sequence of substorm injections from the plasma sheet but rather the result of a sustained and enhanced convection electric field imposed on the magnetosphere. During the storm main phase, most of the ring current magnetic disturbance is due to a partial ring current which closes in part through the ionosphere and in part through the magnetopause. The effect of the enhanced crossmagnetospheric convection electric field is to move Alfvén layers inward with the consequence of further energizing plasma and also moving the ring current closer to the Earth. Only after the enhanced convection electric field is reduced during the storm recovery phase do particles find themselves on closed azimuthal drift paths and the ring current becomes symmetric.

\section{Methodology}

[8] The method of midlatitude magnetic field analysis utilized here follows that described by Clauer et al. [2003]. 


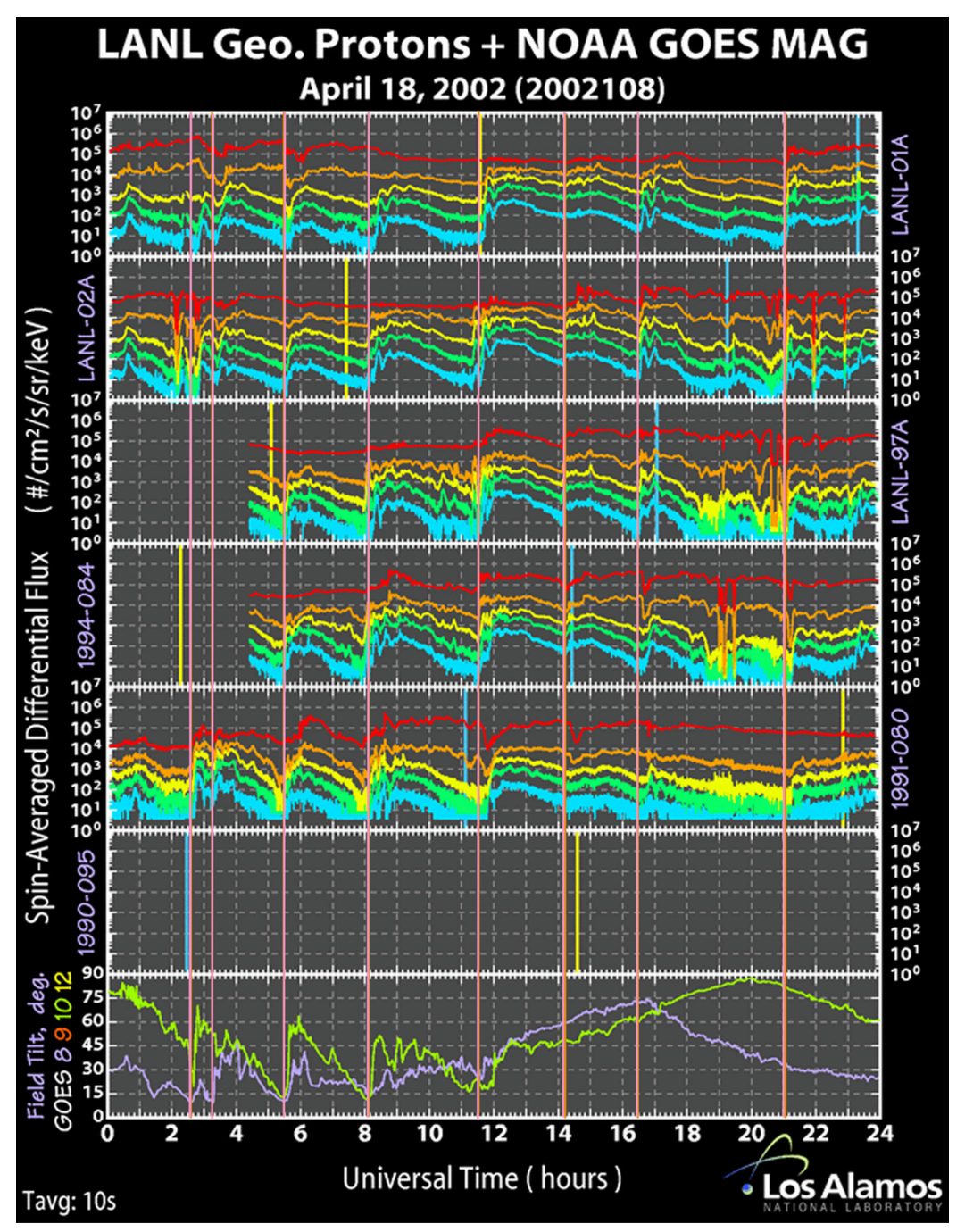

Figure 1. Energetic proton flux data measured at six geostationary satellites (top six panels) and magnetic field tilt angle measured at two geostationary GOES satellites (bottom panel) during 18 April 2002. Vertical yellow bars on the energetic proton flux plots mark the time of local noon at the particular satellite. Vertical blue bars mark the time of local midnight at the satellites. Vertical orange lines mark the onset times of the series of sawtooth oscillations.

The availability of digital data from an extensive chain of midlatitude stations makes possible a computerized analysis of such measurements at high time resolution. Table 1 provides the names and locations in geographic and corrected geomagnetic coordinates of about 27 stations from which high resolution digital data is available during the years 1991 - present. The geomagnetic coordinates calculated in the table are in accordance with Gustafsson et al. [1992]. Note that by assuming the equator to be a plane of symmetry, we may use stations in the southern hemisphere provided that we change the sign of the east component (Y).

[9] We utilize the magnetic data in corrected geomagnetic coordinates, with $\mathrm{X}$ north and $\mathrm{Y}$ east and $\mathrm{Z}$ vertical down. For ring current investigations, we wish to obtain the axial magnetic field perturbation. To obtain the axial perturbation field, we divide the $\mathrm{X}$ component at each station by $\cos \Lambda$ where $\Lambda$ is the corrected geomagnetic latitude of the station. This correction is done primarily for the computation of the $D_{s t}$ index. We do not apply additional data corrections and feel that the data provide sufficiently accurate specification of the three-dimensional current systems for the purposes of this paper. The cos $\Lambda$ correction is done for both disturbed and quiet days. We determine the quiet day variation by selecting several quiet days within 1 month of the disturbed day and the data are averaged.

[10] The quiet day field is subtracted from the data at each station to remove the main field and diurnal variation. The difference between the disturbed and quiet field components then gives a profile of the disturbance field as a function of local time around the world. To create this profile, we linearly interpolate the data between stations to obtain the disturbance value at each minute in local time. The profile is then smoothed using a running average where the averaging window can be specified by the user. In the case presented here, we utilize a 240-min smoothing window. To achieve a periodic profile, we repeat the data at each end of the longitude array and fit the entire array, utilizing the center portion for the local time disturbance profile. 
X Component (Quiet Field Removed)

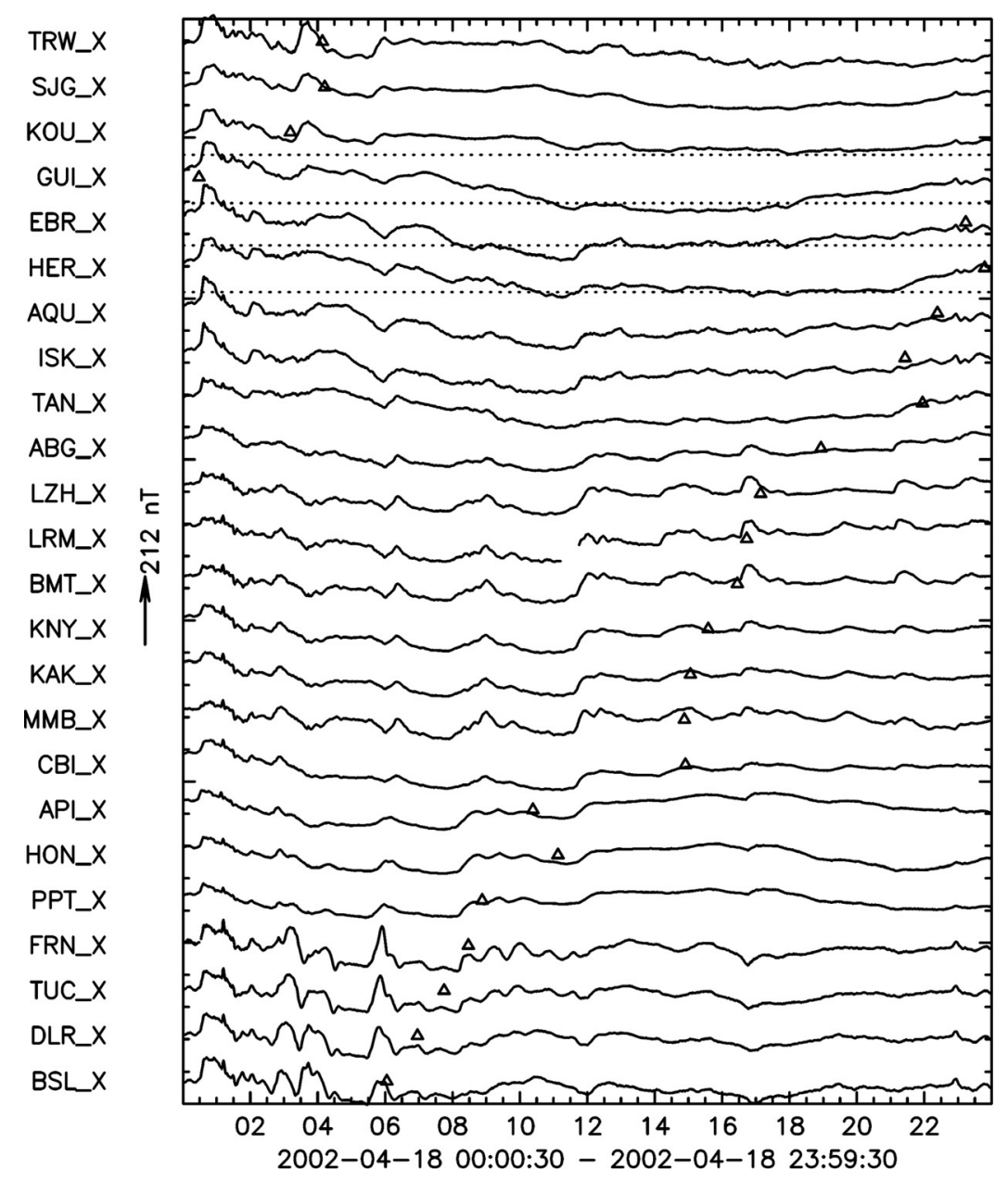

Figure 2. Stack plot of magnetic variation in the $X$ (northward) component of the magnetic field measured on 18 April 2002 by a longitudinal chain of standard observatories. The station names are defined in Table 1. The small triangle above each trace marks local magnetic midnight at each station.

[11] Profiles at successive times can then be plotted in a map as a function of universal time and local time to display the temporal and spatial variation of the midlatitude disturbance field due to substorms and storms. The difference in the $\mathrm{Y}$ component can be investigated for the disturbance variations in the east-west direction. We note that we do not apply a latitude normalization to the Y perturbations because their source is primarily from field-aligned currents and only apply the cosine adjustment to the X component. To minimize latitude effects, we try to limit the observation latitude to lie between $10^{\circ}$ and $45^{\circ}$ (absolute value). A variety of other latitude adjustments can be made with assumptions about the configuration of the disturbance current system, Earth induction, and so forth [Sun et al., 1998; Kitamura et al., 2005]. We have not applied these adjustments. The results of our analysis will provide the general characteristics of the disturbance field. The interpretation of the disturbance field in terms of a substorm current wedge will provide qualitative description that is sufficiently accurate for the purposes of this discussion.

[12] A further benefit of the computer analysis is the ability to easily investigate the changes which occur during a particular interval or phase of a substorm or storm. To isolate a particular magnetic perturbation, we take the local time profile at some reference universal time, for example, the onset time of a sawtooth injection, and then calculate the changes from this profile at successively later universal times. The changes in the local time profiles thus obtained may have simpler form and therefore be easier to understand or model in terms of equivalent currents. Other techniques which can be useful but are not shown here are the use of derivative maps to more accurately determine the time rate of change of the magnetic field. These are obtained by computing the partial derivative of X (or Y) as a function of universal time.

\section{Data Analysis}

[13] The onset times for the 18 April 2002 sawtooth "dipolarizations" are estimated from the energetic proton data measured by Los Alamos Geosynchronous spacecraft. These data are shown in Figure 1. The onset times are estimated to be 0239 UT, 0315 UT, 0530 UT, 0810 UT, 1135 UT, 1407 UT, 1632 UT, and 2104 UT. The proton flux 

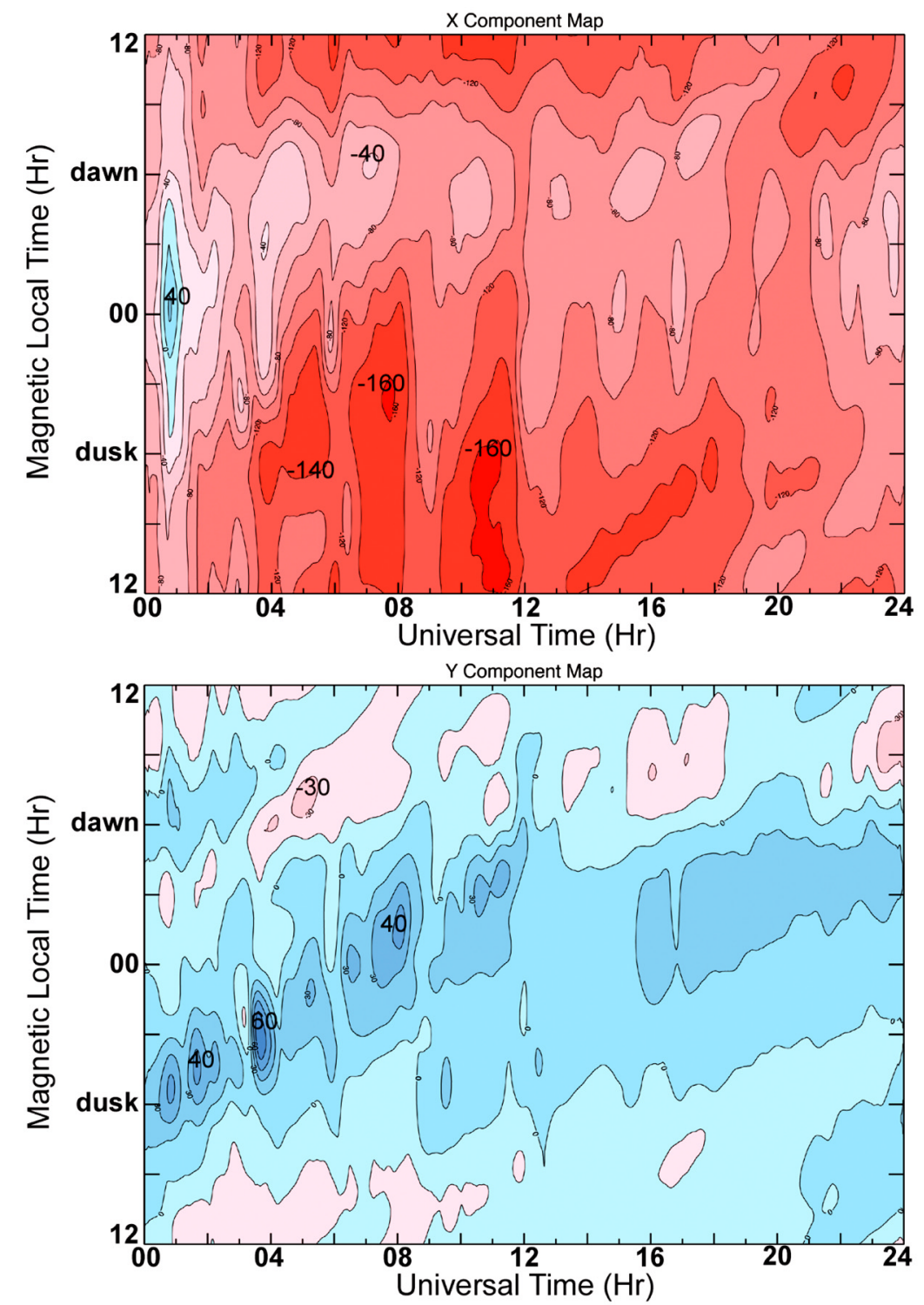

Figure 3. The UT-LT maps of low latitude magnetic perturbations measured on 18 April 2002. X variations (40 $\mathrm{nT}$ contours) are shown in the top map and Y variations (15 $\mathrm{nT}$ contours) are shown in the bottom map. Blue regions indicate positive perturbation and red regions indicate negative perturbation. The vertical axis shows local time with local midnight at center and local noon at the top and bottom edges.

increases are seen globally and the data are consistent with a very broad injection region spanning the nightside and sometimes extending past the dawn or dusk meridians into the dayside magnetosphere. To investigate this further, we examine the temporal and spatial development of the lowlatitude ground magnetic disturbance for several of these sawtooth events and, where data is available, also look at the high-latitude auroral patterns using the UVI instrument on POLAR and the polar ionospheric electrodynamic patterns computed by the AMIE procedure.

[14] Figure 2 shows a stack plot of data from a worldwide longitudinal chain of low latitude magnetic observatories. The traces are arranged according to longitude and a small triangle above each trace indicates the time for local magnetic midnight at each station. We show the X (geo- graphic north) component of the field perturbation (the main field and quiet variation have been removed) at each station.

[15] The storm sudden commencement (SSC) is observed as a jump in the field observed at all stations at about 0030 UT. While it does not have specific bering on the topic discussed here, it is interesting to note that this is an atypical SSC, since the jump magnitude is largest on the nightside rather than near local magnetic noon. For further information on such atypical SSCs, see Clauer et al. [2001] and Belenkaya et al. [2004]. Following the SSC, there is a set of periodic variations over a large portion of the stations with a 3-4 hour period. We can display these data in another form to better see both the temporal and spatial variations. This is shown in Figure 3. The top map in Figure 3 shows the northward axial component $\mathrm{X}$ (the same data shown in the 


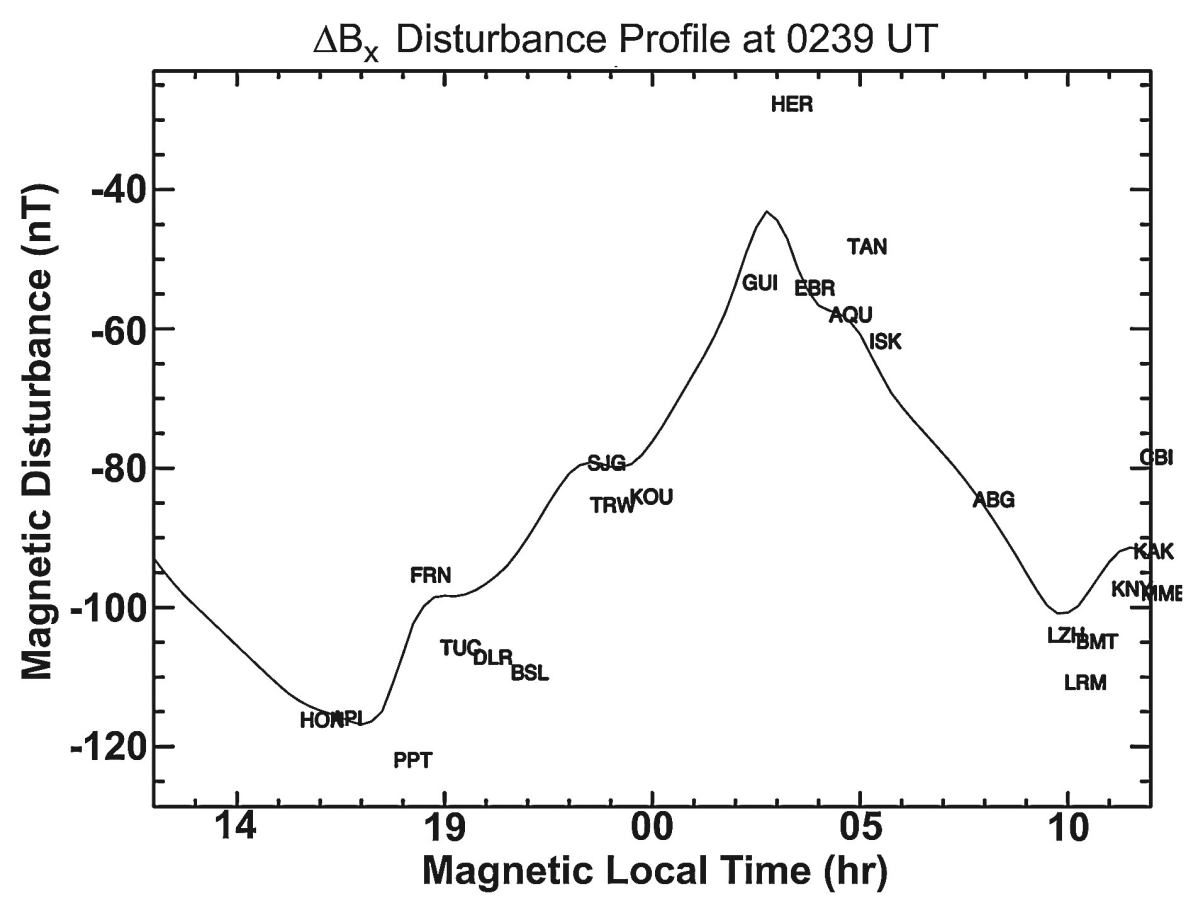

Figure 4. Magnetic disturbance profile at 0239 measured in the $\Delta \mathrm{X}$ component. Three letter codes denote station names from Table 1 displayed at the local time and measurement value for each station used to create the profile. The quiet time main field has been removed as discussed in the text.

stackplot in Figure 2) and the bottom map shows the eastward component Y in LT-UT map format. The vertical axis shows local time with local magnetic midnight at the center and noon at the top and bottom edges. Negative field perturbation is shown by red shaded contours and positive perturbation is shown by blue shaded contours. The disturbances that appear in the top X map are due primarily to the asymmetric and symmetric ring current, the substorm current wedge, and currents responsible for the atypical SSC at 0030 UT. The perturbations shown in the bottom Y map can be used to identify the locations of fieldaligned currents with positive perturbation indicating outward FAC and negative perturbation indicating inward FAC.

[16] Removal of the main field and quiet variation is a process subject to error because of the variability of the various currents that contribute to the quiet time field, for example, Sq currents, quiet tail, and magnetopause currents, etc. [Clauer et al., 1980]. There is also a diagonal trend in the data which matches the station motion across the map. We continue to investigate this but suspect that it results because peaks in the disturbance profile are observed by stations in a specific region, but they rotate with time carrying the peak in the profile with them. While we continue to seek a solution to this problem, we can eliminate much of this effect for short intervals of time by selecting a reference time and subtracting the data values at the reference time from all later values for each station. These "referenced" values are then used to create the local time profiles that are contoured to form the "referenced" UT-LT map. Thus we can show with less error, the development of magnetic perturbations following some reference time. In the case here, we can utilize the onset time of each sawtooth and then create maps of the perturbations referenced to the onset time.
[17] Figure 4 shows the $\mathrm{X}$ component magnetic disturbance profile at 0239 UT obtained from the map shown in Figure 3. The horizontal axis is local time position around the Earth and the vertical axis is the magnetic disturbance measured in nanotesla. Also shown are the stations and measurement values used to create the profile. This presents the distribution of stations in local time and the typical scatter in data values that are fit. Consistent with the ongoing magnetic storm, the field is depressed but highly asymmetric, with the greatest depression near dusk $-118 \mathrm{nT}$ and only $-47 \mathrm{nT}$ at 0230 MLT.

[18] Figure 5 shows the $\mathrm{X}$ and $\mathrm{Y}$ maps referenced to the field at 0239 UT. That is, the profile at 0239 UT has been subtracted from the data that are displayed in the maps. For the times following 0239 UT we see the development of magnetic perturbations due to the changes in current distributions following the sawtooth onset at 0239 UT. We note that the 0239 UT and 0315 UT onsets are closely spaced and in this analysis consider them together as a double-onset event. The top shows the $\Delta X$ field perturbations and these exhibit the classic positive bay signature centered near local midnight. Such a signature is usually associated with the substorm expansion phase. The first expansion onset is centered near 2000 MLT and reaches a peak at about 0304 UT and begins a small recovery prior to the second onset at 0315 UT. The second expansion is centered near 2300 MLT and reaches a peak at 0341 UT. We define the local time extent as the width of the positive $\Delta X$ profile at half maximum. The first expansion is narrow measuring only about 3.25 hours in MLT extent and the 0304 UT peak. The second expansion is broader and measures about 5 hours in local time extent at 0341 UT.

[19] From the $\Delta Y$ component map we see the formation of a strong field-aligned currents bounding both expansions 

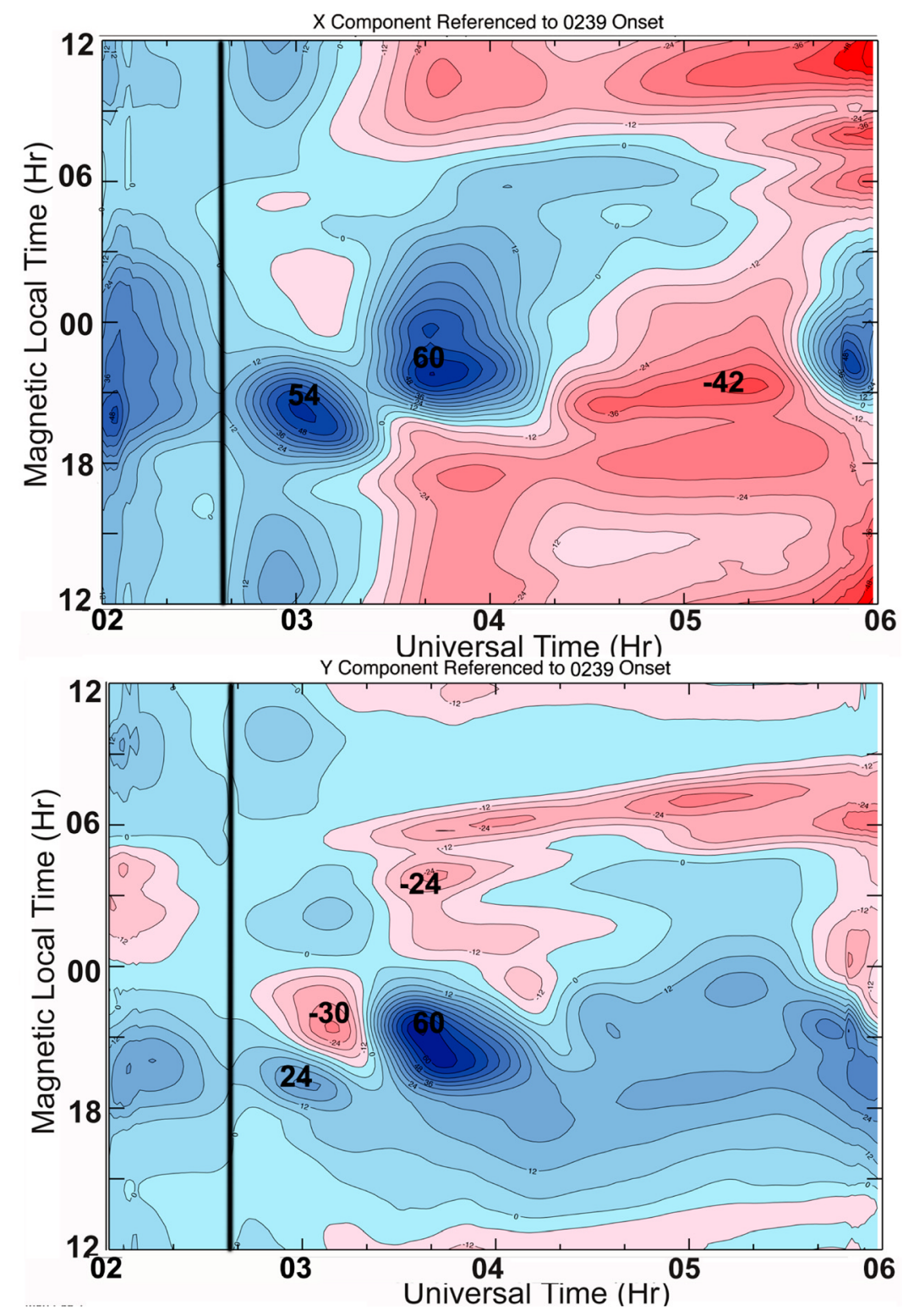

Figure 5. Maps of magnetic perturbation referenced to the sawtooth onset at 0239 UT. The contour interval in both maps is $6 \mathrm{nT}$. The top map shows the $\Delta \mathrm{X}$ component and the bottom map shows the $\Delta \mathrm{Y}$ component. Positive $\Delta \mathrm{Y}$ signifies the location of an outward field-aligned current while negative $\Delta \mathrm{Y}$ signifies the location of inward field-aligned current.

observed in $\Delta X$ with inward current (negative $\Delta Y$ contours) on the dawnward side and outward current (positive $\Delta Y$ contours) on the duskward side of the system.

[20] We have examined the low-latitude ground magnetic disturbance for each of the next three individual sawteeth, referencing the disturbance field to the field a the time of the sawtooth onset, similar to the procedure shown in Figure 5. The results of this are shown in the sequence of plates from Figures 6 to 8 .

[21] Figure 6 shows $\Delta X$ and $\Delta Y$ maps in a format similar to Figure 9 . In addition, however, we have also added highlatitude data at select times that are noted on the maps by vertical lines. The five high-latitude plots along the top of the figure combine UV images of auroral emissions obtained from the Polar satellite UVI instrument with electric potential patterns determined using the Assimilative Mapping of Ionospheric Electrodynamics (AMIE) [c.f. Richmond and Kamide, 1988; Richmond, 1992] procedure utilizing an array of high-latitude magnetic observations. The first pattern is at 0528 UT just prior to the sawtooth onset. It shows a DP2-like electric potential patterns and auroral emissions near dawn in the convection electrojet region. The second pattern is at the time of sawtooth dipolarization onset, $0530 \mathrm{UT}$. The potential pattern is very similar to the earlier DP2 pattern. In the auroral emissions, however, we see an onset of intensification near 2300 MLT, just prior to local midnight. In the following three panels we see this premidnight auroral intensification grow and expand. The expansion is similar to that generally associated with a magnetospheric substorm. Near the peak of the 

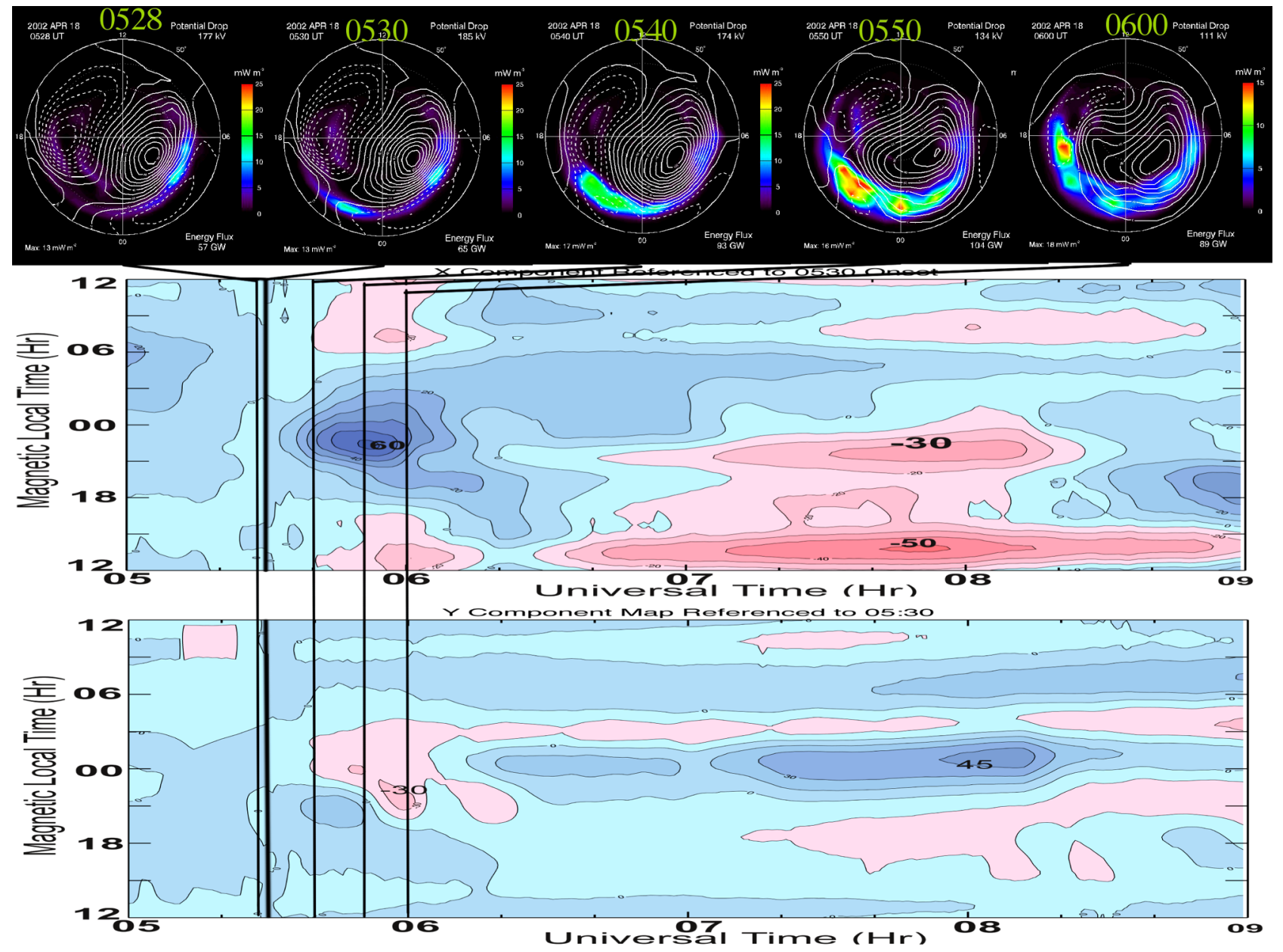

Figure 6. $\Delta \mathrm{X}$ and $\Delta \mathrm{Y}$ maps of magnetic perturbation referenced to the sawtooth onset at $0530 \mathrm{UT}$. Contours are drawn at $10 \mathrm{nT}$ intervals in both maps. Along the top of the plot are polar potential patterns derived from AMIE analysis of high-latitude global magnetometer measurements and superposed UV auroral images from the Polar satellite at 0528, 0530, 0540, 0550, and $0600 \mathrm{UT}$.

expansion, $0550 \mathrm{UT}$, the extent is rather broad from dawn to dusk, and the potential pattern has rotated with the positive cell becoming dominate, similar to the typical DP1-type substorm pattern. At 0600 UT during the recovery, the pattern has expanded further in local time and also poleward in latitude. This behavior is consistent with the unloading of energy stored in tail lobes.

[22] From the UT-LT maps we see a positive perturbation near local midnight in the $\Delta X$ component, similar to that shown in Figure 5. There is no enhancement of the partial ring current associated with the positive perturbations that develop at 0530 UT. The local time extent of the positive perturbation is more narrow than the previous sawtooth, being about 3.8 hours in MLT. The field-aligned currents inferred from the $\Delta Y$ component map are very weak, suggesting that both the downward and upward FAC are very diffuse.

[23] Turning to the 0810 UT sawtooth onset, Figure 7 shows again a combination of high-latitude and low-latitude data. The UT-LT maps of the low-latitude magnetic observations referenced to 0810 , prior to the sawtooth onset, show a broader and somewhat weaker $\Delta X$ perturbation. The extent stretches from near 0000 MLT to 1600 MLT. Again there is no simultaneous enhancement of the partial ring current with the positive perturbations that begin at $0810 \mathrm{UT}$. There is a broad downward field-aligned current centered near local midnight and broad, diffuse upward field-aligned current elsewhere indicated by the $\Delta Y$ map.

[24] The high-latitude data show characteristics very similar to the 05:30 UT onset in Figure 6. Just prior to the onset at $0810 \mathrm{UT}$, the potential patterns is a two-cell DP2 convection pattern with auroral emission enhancement near dawn in the convection electrojet. At 0820 UT during the expansion phase, the electrojet emissions have intensified across the nightside. The extent of the enhanced emissions is very broad extending from dawn through the nightside past dusk. The positive potential cell becomes more dominate and rotates to become oriented more like a DP1 pattern; however, it seems to be intermediate between the typical DP1 and DP2 patterns. The auroral emissions are weaker than the previous sawtooth event and somewhat more patchy around the auroral oval. The strongest intensification in the auroral emissions appears to be at dusk. The auroral emissions have also moved poleward at 0901 suggesting a decrease in the polar caps size.

[25] The 1135 UT sawtooth perturbations in the UT-LT maps show similar features in Figure 8. High-latitude UV 


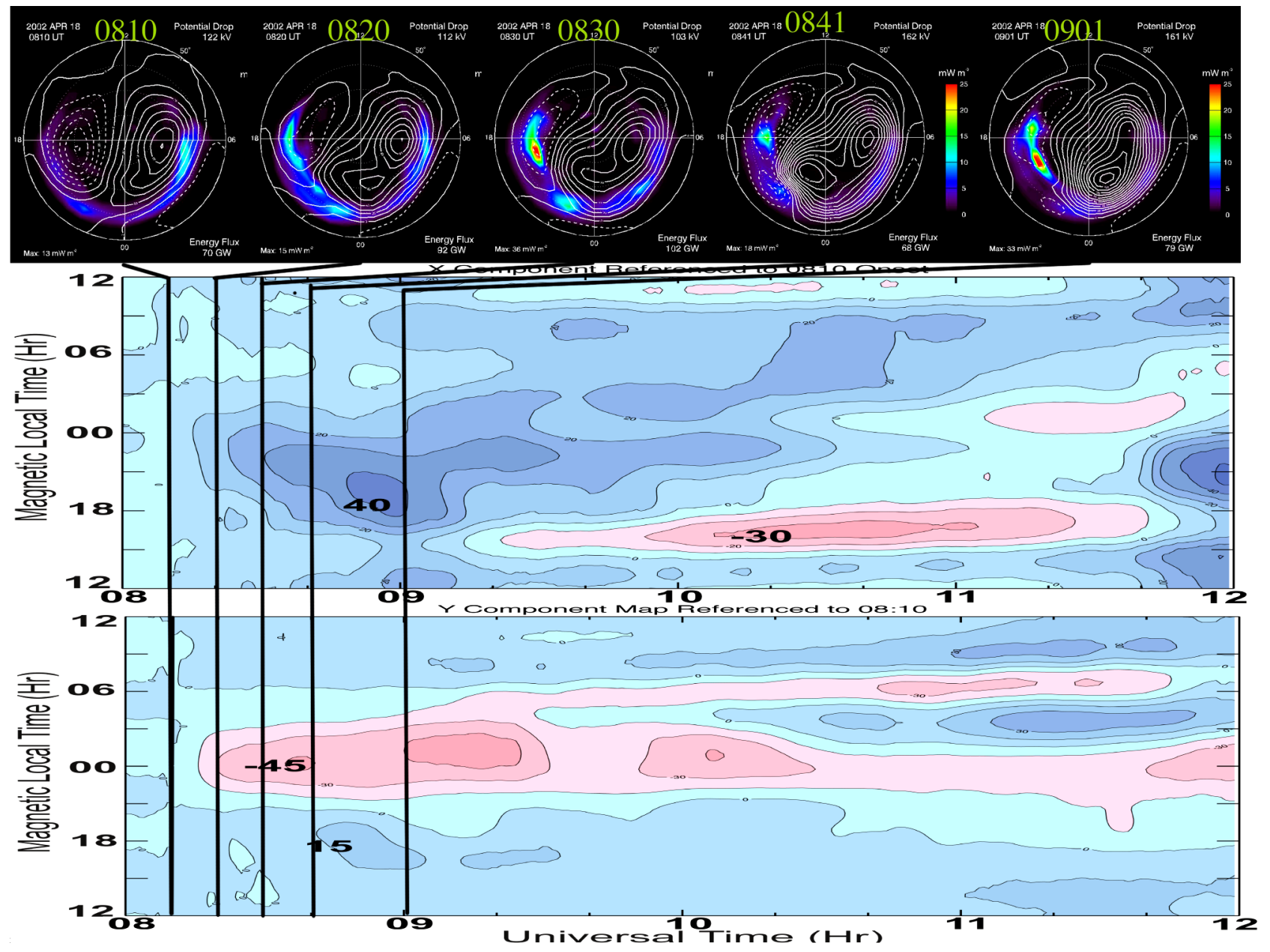

Figure 7. $\Delta \mathrm{X}$ and $\Delta \mathrm{Y}$ maps of magnetic perturbation referenced to the sawtooth onset at $0810 \mathrm{UT}$. The contour level for the $\Delta \mathrm{X}$ map is $10 \mathrm{nT}$ and for the $\Delta \mathrm{Y}$ map is $15 \mathrm{nT}$. Along the top of the plot are polar potential patterns derived from AMIE analysis of high-latitude global magnetometer measurements and superposed UV auroral images from the Polar satellite at 0810, 0820, 0830, 0841, and 0901 UT.

data from Polar are not available during this event so we show only the low-latitude magnetic maps. The perturbations are somewhat weaker but broader in extent in the $\Delta X$ map. Inward field-aligned current (negative perturbations in the $\Delta Y$ map) flow along the edge of the $\Delta X$ positive enhancement at 0300 MLT. The upward field-aligned current is again diffuse but seems to lie along the dusk edge of the $\Delta \mathrm{X}$ positive bay.

[26] There is considerable contrast between each of the sawteeth. The extent of the positive bay disturbance, magnitude, and central meridian in the low-latitude magnetic disturbance profile for each sawtooth is different. The profiles near the peak of the magnetic disturbance for each of the four sawteeth discussed above are shown in Figure 9. We measure the local time extent of the substorm disturbance profile as the full width at half maximum. The local time extent ranges from 12 hours, which is much larger than typical isolated substorms, to a little over 3 hours, which is common for typical isolated substorms. The $\Delta \mathrm{X}$ profiles also show considerable variability in the local time location of the central meridian as well. The first (0304 UT), fourth (0852 UT), and fifth (1220 UT) $\Delta \mathrm{X}$ profiles show the disturbances centered several hours premidnight while the second (0341 UT) and third (0550 UT) profiles are centered near local midnight.

[27] G. D. Reeves et al. (Substorm injection boundaries during the April 18, 2002 sawtooth event and dayside dispersionless injections, manuscript in preparation, 2006, hereinafter referred to as Reeves et al., manuscript in preparation, 2006) investigate the energy dispersion in the energetic proton fluxes shown in Figure 1 to estimate the location and extent of the region over which the injection is dispersionless, the injection boundary. In Table 2 we contrast the magnetic profile parameters against the injection boundary parameters obtained by Reeves.

[28] Comparison of the local time magnetic disturbance profile parameters of the local time injection boundary parameters, there appears to be little relationship between the injection boundary at the onset of the disturbance with the substorm current wedge and associated low-latitude ground disturbance field at the peak of the disturbance (end of the expansion). This might also be expected since at onset; generally the auroral signature is a very localized brightening that expands both poleward and in longitude. The auroral disturbance and low-latitude magnetic disturbance are generally more localized than the injection 

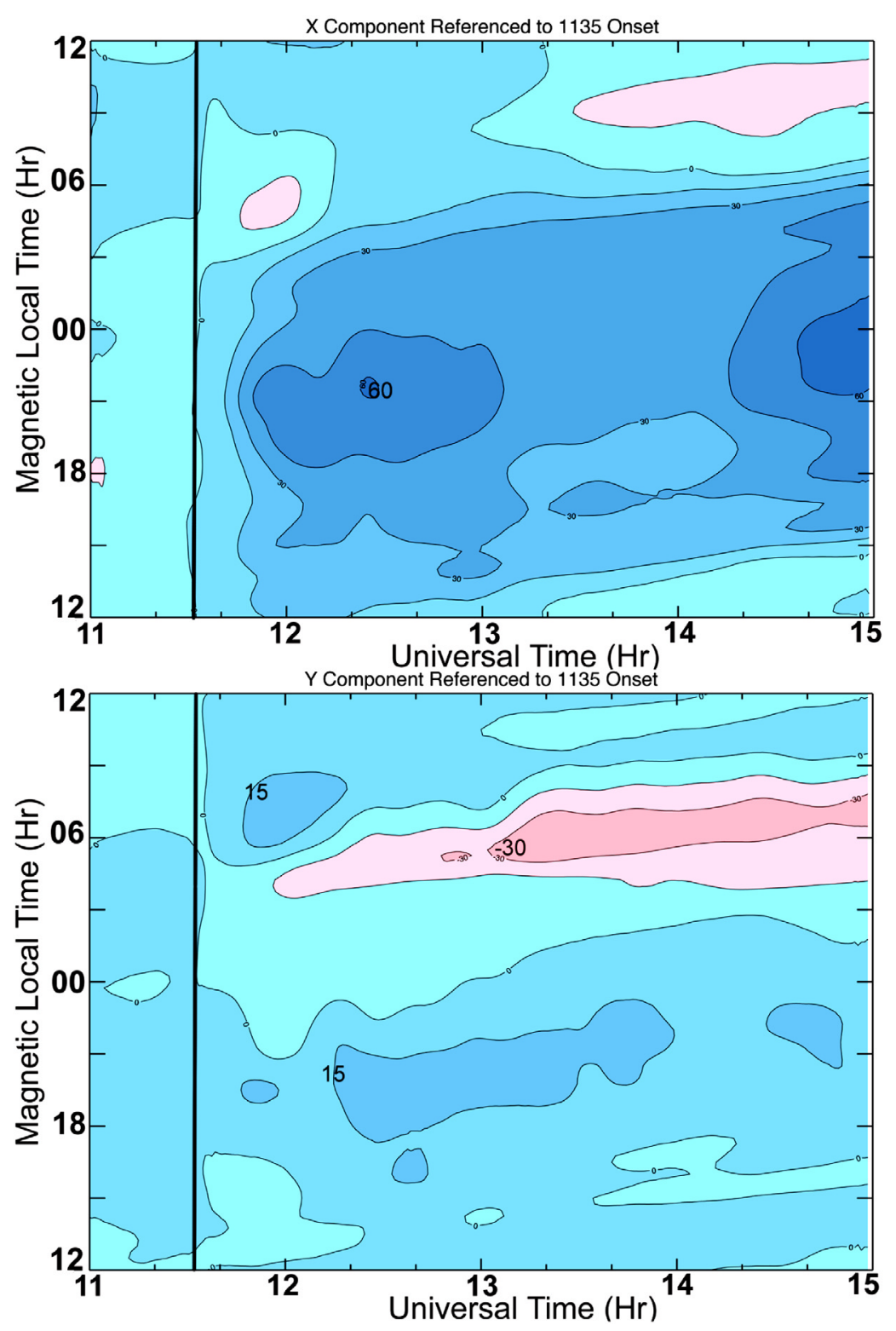

Figure 8. $\Delta \mathrm{X}$ and $\Delta \mathrm{Y}$ maps of magnetic perturbation referenced to the sawtooth onset at $1135 \mathrm{UT}$. The contour level for both maps is $15 \mathrm{nT}$.

boundary; however, the opposite appears to be the case for the 1135 UT sawtooth.

\section{Discussion}

[29] Huang et al. [2003] examined a number of sawtooth injection events and concluded that they were simply periodic substorms. The flux dropout is related to the tail stretching during the growth phase of the substorm and the flux increase represents particle injection to geosynchronous orbit from the tail as the field undergoes dipolarization. All of the data signatures examined in the tail during sawtooth events were unremarkable and consistent with typical substorm expansions. The low-latitude ground signatures are largely consistent with substorm development, as well. However, the magnetic disturbance of the substorm current wedge is sometimes much broader in local time extent than typical isolated substorms [Clauer and McPherron, 1974b].
The average local time extent for isolated substorms from an examination of 41 events was 2.5 hours in MLT extent compared to the approximate 7.7-hour average MLT extent measured for the sawtooth events examined here. More recent studies of isolated substorms using a denser longitudinal chain of observatories obtains an average local time extent of the substorm current wedge to be about 4 hours in MLT (D. T. Welling, personal communication, 2005). The injection boundaries determined by Reeves et al. (manuscript in preparation, 2006) extend across the nightside and in several cases past the terminators to the dayside. This distinguishes the sawtooth events from the more localized injection region found during typical isolated substorms. The magnetic disturbance profiles measured at low latitudes are comparable or larger to the disturbance profiles determined for typical isolated substorms. In terms of the magnetic disturbance, there is little to distinguish sawtooth events from substorm expansions. As seen in Figure 3, 
Local Time Profiles of Magnetic Disturbance

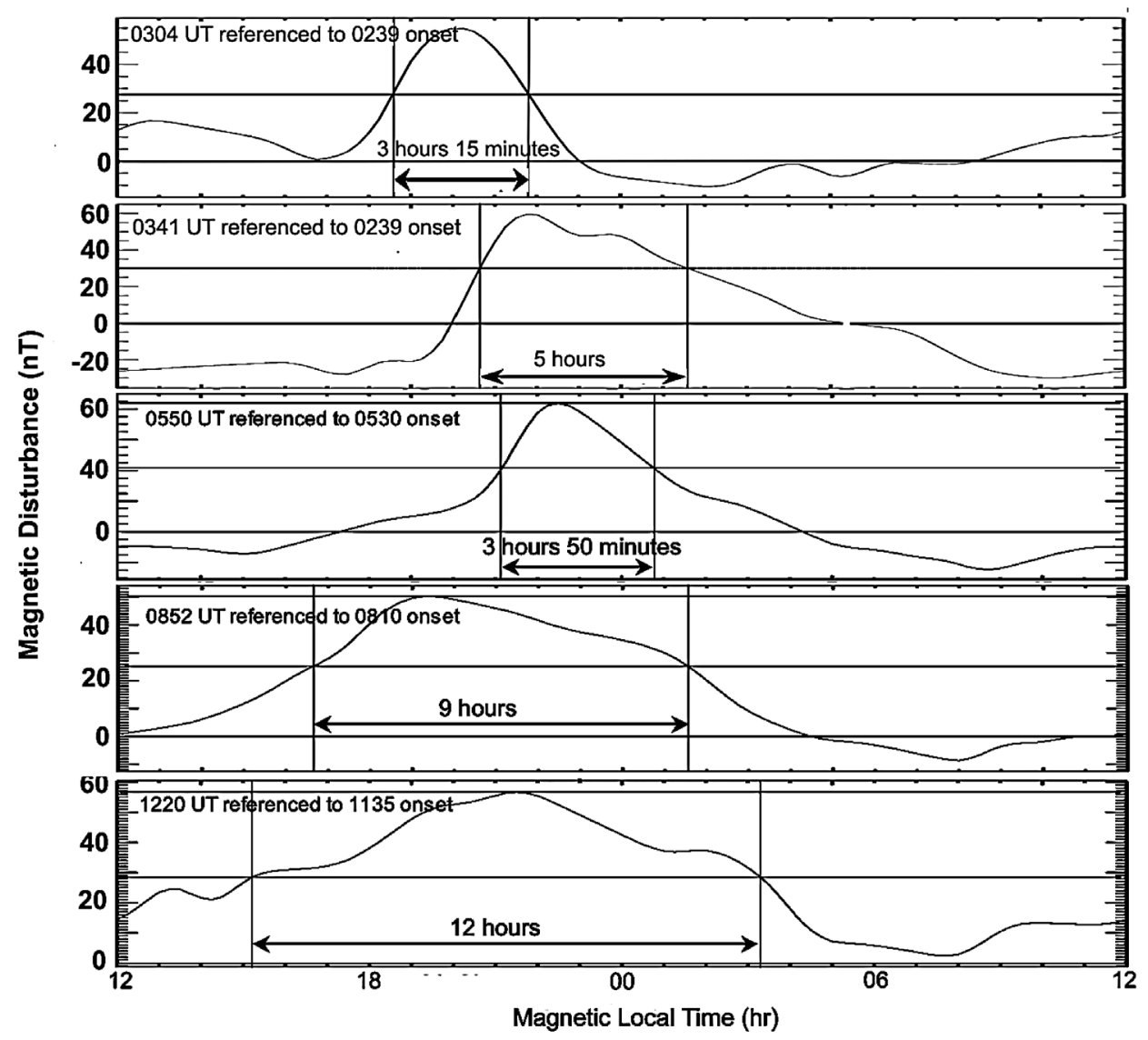

Figure 9. Magnetic local time profiles of the magnetic disturbance near the peak of the four sawtooth events at (starting from the top) with onset at 0229 UT (and double peaks at 0304 UT and 0341 UT), 0530 UT, 0810 UT, and 1135 UT. The magnetic disturbance profile at the onset time has been subtracted from the profile displayed here near the peak of each event.

sawtooth events develop during an interval that has already produced a strong partial ring current. There is little additional development of the asymmetric ring current produced by each of the successive sawtooth event.

[30] Kitamura et al. [2005] examined the 18 April 2002 sawtooth events and also concluded that morphologically, sawtooth events appear very similar to substorms; however, they are larger than normal in their local time extent. The magnetic disturbance is consistent with the substorm current wedge and the initiation of the sawtooth expansion is heralded by $\mathrm{Pi} 2$ pulsations with polarization characteristics also consistent with typical substorm expansions, albeit with wider local time extent than typical. In other studies of sawtooth events as well, the conclusion ranks these disturbances as indistinguishable form substorms except that the disturbances are wider than is typical of most substorms [Reeves et al., 2003; Henderson, 2004]. In terms of the current system, particle injection, tail stretching and dipolarization, PI2 generation, etc. we must agree that sawtooth events have all of the characteristics of substorms and should probably be regarded as a class of substorm phenomena.

[31] Borovsky et al. [1993] distinguished periodic substorms from sawtooth events, based largely on the local time extent of the disturbance, and found that periodic substorms occur generally during intervals of high-speed solar wind

Table 2. Magnetic Disturbance Parameters Versus Energetic Flux Injection Parameters ${ }^{\mathrm{a}}$

\begin{tabular}{|c|c|c|c|c|c|c|c|}
\hline \multicolumn{4}{|c|}{ Magnetic Profile Parameters (This Paper) } & \multicolumn{4}{|c|}{ Particle Flux Injection Parameters (Reeves) } \\
\hline $\begin{array}{l}\text { Profile Time, } \\
\text { UT }\end{array}$ & $\begin{array}{l}\text { Dusk Bound, } \\
\text { MLT }\end{array}$ & $\begin{array}{c}\text { Dawn Bound, } \\
\text { MLT }\end{array}$ & $\begin{array}{c}\text { Extent, } \\
\text { hours }\end{array}$ & $\begin{array}{c}\text { Injection Time, } \\
\text { UT }\end{array}$ & $\begin{array}{l}\text { Dusk Bound, } \\
\text { MLT }\end{array}$ & $\begin{array}{c}\text { Dawn Bound, } \\
\text { MLT }\end{array}$ & $\begin{array}{c}\text { Extent, } \\
\text { hours }\end{array}$ \\
\hline 0304 & 1840 & 2155 & 3.25 & 0240 & 1500 & 0100 & 10 \\
\hline 0341 & 2040 & 0140 & 5. & 0240 & 1500 & 0100 & 10 \\
\hline 0550 & 2105 & 0045 & 3.67 & 0532 & 1400 & 0700 & 17 \\
\hline 0852 & 1640 & 0140 & 9 & 0756 & 1400 & 0100 & 11 \\
\hline 1220 & 1510 & 0310 & 12 & 1120 & 1800 & 0100 & 7 \\
\hline
\end{tabular}

${ }^{a}$ The boundaries of the injection are the minimum determined by Reeves et al. (manuscript in preparation, 2006). 


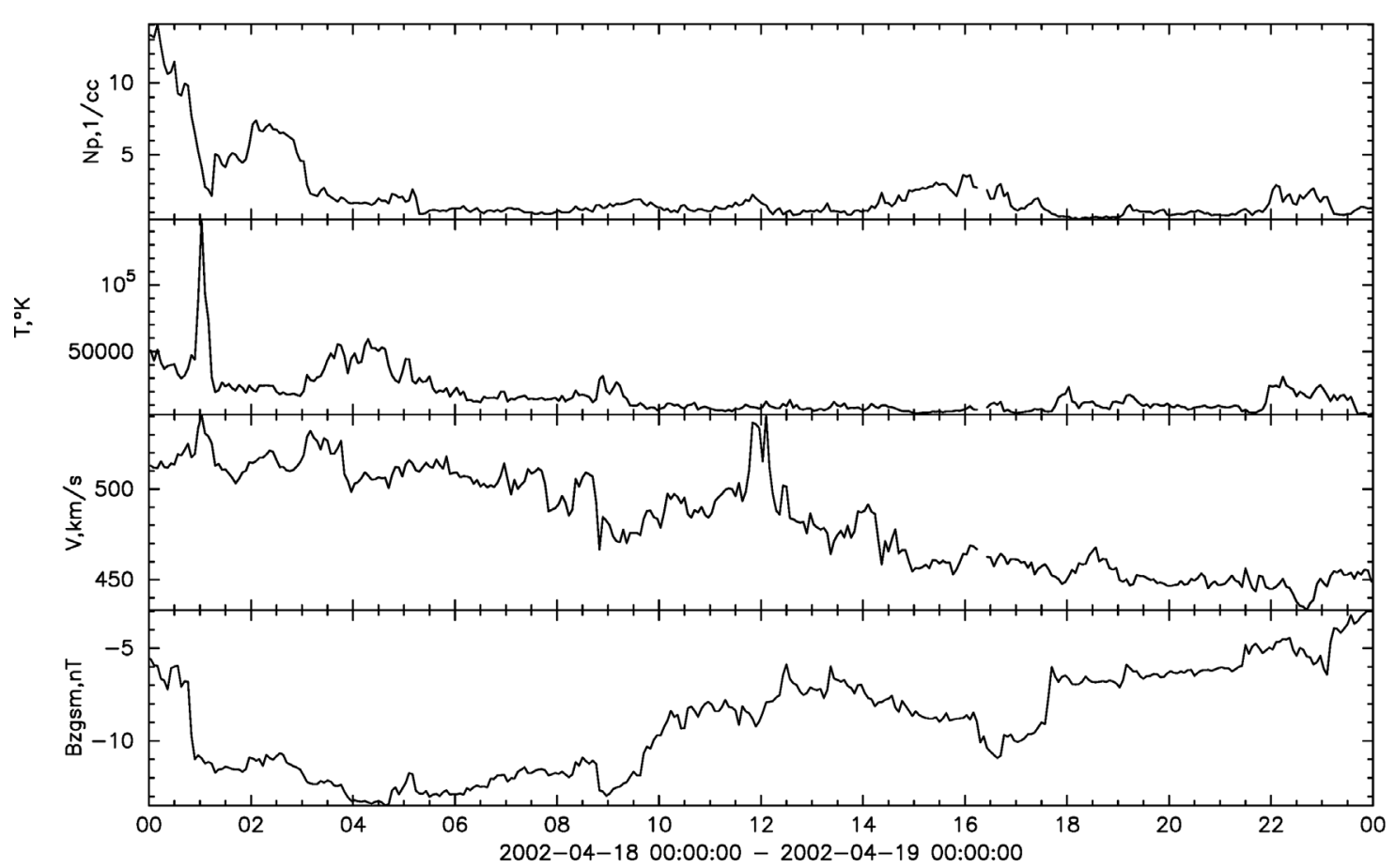

Figure 10. From the top, solar wind proton number density, temperature, bulk velocity, and the IMF $B_{z}$ component in gsm coordinates measured at the L1 point by the ACE satellite.

streams. During these intervals there are frequent short periods of IMF southward $B_{z}$ that gradually produce an accumulation of magnetic energy in the tail that is released at each substorm expansion. Sawtooth events, on the other hand, generally occur during strong steady driving. The 18 April 2002 events occur during a magnetic storm that is driven by a magnetic cloud which implies a slowly changing IMF. Figure 10 shows the IMF $B_{z}$ component along with solar wind velocity and number density and temperature measured by the ACE satellite at the L1 location upstream. The ACE data have not be time shifted to account for the propagation time from L1 to the subsolar magnetosphere. The propagation time is about 1 hour and the sawtooth events occur during the passage of a magnetic cloud. The characteristic cloud signatures include the low number density and temperature and slow rotation of the magnetic field. The IMF Bz component is negative around $-8 \mathrm{nT}$ for most of the sawtooth interval. Such quasi-stable condition often produce steady magnetic convection intervals when the magnetic reconnection rate in the tail adjusts to match the dayside merging rate.

[32] The driving conditions during the period of sawtooth oscillations should produce a moderately strong, continuous driving. The variability of the driving conditions is relatively small and during other events, similar quasi-steady conditions lead to periods of steady magnetospheric convection. Thus a major issue to resolve is the conditions that lead to either sawtooth oscillations or steady magnetospheric conditions. We suggest that in the case of sawtooth oscillations, some internal threshold must be exceeded that prevents steady convection and energy dissipation and this then leads to the global sawtooth oscillations. In the "loadingunloading" phenomenological model of the magnetospheric substorm, it is the imbalance of the dayside and nightside merging rates that results in an accumulation of magnetic energy in the tail lobes that is then suddenly released by a the formation of a new near-Earth neutral line and enhance tail reconnection during the expansion phase. This is certainly the case during the periodic substorm events observed during high-speed streams. There is considerable evidence that when the IMF turns southward (enhance dayside merging) and remains stable for an extended period of time, the magnetosphere can adjust to balance dayside and nightside reconnection thus resulting in enhanced but steady magnetospheric convection [Pytte et al., 1978; Sergeev et al., 1996]. This is frequently the case during magnetic storms. However, we see here that during some storm intervals, sawtooth oscillations may develop rather than steady magnetospheric convection. It remains to determine the conditions that will produce either SMC or sawtooth events during quasi-steady enhanced magnetospheric driving. Sawtooth events show many features of the substorm phenomena, including a decrease in polar cap size following the expansion. This signature has been attributed to unloading of magnetic energy flux that has accumulated in the tail lobes, generally as a consequence of an imbalance between the dayside and nightside reconnection rates brought about by an increase in the dayside reconnection rate. In general, it has been assumed that variability of the dayside reconnection rate is the primary cause of imbalance and the subsequent phenomenology that leads to a substorm expansion onset. In sawtooth events, however, the driver is steady and it is necessary to examine the internal magnetospheric dynamics to understand what prevents the tail reconnection rate from increasing to match the dayside rate and instead, produce global substormlike periodic oscillations. A necessary step toward understanding these phenomena will be a statistical investigation to determine the distribution of driving conditions and magnetospheric conditions that exist during each of these stormtime phenomena. 
[33] Acknowledgments. Much of this work was initiated and developed as a result of the Taos Sawtooth Workshops sponsored by the Los Alamos National Laboratory Institute of Geophysics and Planetary Physics. Support for the work at the University of Michigan came from NASA grants NAG5-12176 and NAG5-12040 and a grant from the National Science Foundation OPP-0220735. The AMIE potential plots and superposed auroral UVI data were provided by Gang Lu. We also thank Geoff Reeves for productive discussions and for sharing the results of his data and analysis of injection boundaries with us.

[34] Arthur Richmond thanks Chao-Song Huang and another reviewer for their assistance in evaluating this paper.

\section{References}

Belenkaya, E. S., I. I. Alexeev, and C. R. Clauer (2004), Field-aligned current distributions in the transition current system, J. Geophys. Res. 109, A11207, doi:10.1029/2004JA010484.

Borovsky, J., R. J. Nemzek, and R. D. Belian (1993), The occurrence rate of magnetopheric-substorm onsets: Random and periodic substorms, J. Geophys. Res., 98, 3807-3813.

Clauer, C. R., and R. L. McPherron (1974a), Mapping the local timeuniversal time development of magnetospheric substorms using midlatitude magnetic observations, J. Geophys. Res., 79, 2811.

Clauer, C. R., and R. L. McPherron (1974b), Variability of midlatitude magnetic parameters used to characterize magnetospheric substorms, J. Geophys. Res., 79, 2898-2900.

Clauer, C. R., and R. L. McPherron (1980), The relative importance of the interplanetary electrric field and magnetospheric substorms on partial ring current development, J. Geophys. Res., 85, 6747.

Clauer, C. R., R. L. McPherron, and M. G. Kivelson (1980), The uncertainty in ring current parameters due to the quiet magnetic field variability at midlatitudes, J. Geophys. Res., 85, 633-643.

Clauer, C. R., I. I. Alexeev, E. S. Belenkaya, and J. B. Baker (2001), Special features of the 24-27 September, 1998 storm during high solar wind dynamic pressure and northward IMF, J. Geophys. Res., 106, $25,695-25,711$

Clauer, C. R., M. W. Liemohn, J. U. Kozyra, and M. L. Reno (2003), The relationship of storms and substorms determined from midlatitude ground-based magnetic maps, in Disturbances in Geospace: The Storm-Substorm Relationship, vol. 142, edited by A. S. Sharma, Y. Kamide, and G. S. Lakhina, pp. 143-157, AGU, Washington, D.C.
Gustafsson, G., N. E. Papitashvili, and V. O. Papitashvili (1992), A revised corrected geomagnetic corrdinate system for epoch 1985 and 1990 J. Atmos. Terr. Phys., 54, 1609.

Henderson, M. G. (2004), The May 2-3, 1986 CDAW-9C interval: A sawtooth event, Geophys. Res. Lett., 31, L11804, doi:10.1029/2004GL019941. Huang, C.-S., J. C. Foster, G. D. Reeves, G. Le, H. U. Frey, C. J. Pollock, and J.-M. Jahn (2003), Periodic magnetospheric substorms: Multiple space-based and ground-based instrumental observatiosn, J. Geophys. Res., 108(A11), 1411, doi:10.1029/2003JA009992.

Kamide, Y., and N. Fukushima (1971), Analysis of magnetic storms with DR-indices for equatorial ring current field, Rep. Ionos. Space Res. Jpn., $25,125$.

Kitamura, K., H. Kawano, S. Ohtani, A. Yoshikawa, and K. Yumoto (2005), Local-time distribution of low and middle latitude ground magnetic disturbances at sawtooth injections of April 18-19, 2002, J. Geophys. Res., 110, A07208, doi:10.1029/2004JA010734.

Pytte, T., R. L. McPherron, E. W. Hones Jr., and H. I. West Jr. (1978), Multiple-satellite studies of magnetospheric substorms: Distinction between polar magnetic substorms and convection-driven negative bays, J. Geophys. Res., 83, 663.

Reeves, G. D., et al (2003), Image, polar, and geosynchronous observations of substorm and ring current ion injection, in Disturbances in Geospace: The Storm-Substorm Relationship, vol. 142, edited by A. S. Sharma, Y. Kamide, and G. S. Lakhina, pp. 91-101, AGU, Washington, D.C.

Richmond, A. D. (1992), Assimilative mapping of ionospheric electrodynamics, Adv. Space Res., 12, 59.

Richmond, A. D., and Y. Kamide (1988), Mapping electrodynamic features of the high-latitude ionosphere from localized observations: Technique, J. Geophys. Res., 93, 5741-5759.

Sergeev, V., R. J. Pellinen, and T. I. Pulkinnen (1996), Steady magnetospheric convection: A review of recent results, Space Sci. Rev., 75, 551.

Sun, W., W.-Y. Xu, and S.-I. Akasofu (1998), Mathematical separation of directly driven and unloading components in the ionospheric equivalent currents during substorms, J. Geophys. Res., 103, 11,695.

X. Cai, C. R. Clauer, A. DeJong, and D. Welling, Space Physics Research Laboratory, University of Michigan, 2455 Hayward, Ann Arbor, MI 481092143, USA. (bob.clauer@umich.edu)

M. G. Henderson, Los Alamos National Laboratory, ISR-1, Mail Stop D466, Los Alamos, NM 87545, USA. 